ARTICLE

\title{
A single atom change turns insulating saturated wires into molecular conductors
}

\author{
Xiaoping Chen ${ }^{1,2}$, Bernhard Kretz (i) ${ }^{3}$, Francis Adoah ${ }^{4}$, Cameron Nickle ${ }^{4}$, Xiao Chi ${ }^{5}$, Xiaojiang Yu (i) ${ }^{5}$, \\ Enrique del Barco (1) ${ }^{4}$, Damien Thompson (1) ${ }^{6}$, David A. Egger ${ }^{3 凶}$ \& Christian A. Nijhuis (1) 1,2,7凶
}

We present an efficient strategy to modulate tunnelling in molecular junctions by changing the tunnelling decay coefficient, $\beta$, by terminal-atom substitution which avoids altering the molecular backbone. By varying $\mathrm{X}=\mathrm{H}, \mathrm{F}, \mathrm{Cl}, \mathrm{Br}$, I in junctions with $\mathrm{S}\left(\mathrm{CH}_{2}\right)_{(10-18)} \mathrm{X}$, current densities $(J)$ increase $>4$ orders of magnitude, creating molecular conductors via reduction of $\beta$ from 0.75 to $0.25 \AA^{-1}$. Impedance measurements show tripled dielectric constants $\left(\varepsilon_{\mathrm{r}}\right)$ with $X=I$, reduced HOMO-LUMO gaps and tunnelling-barrier heights, and 5 -times reduced contact resistance. These effects alone cannot explain the large change in $\beta$. Densityfunctional theory shows highly localized, X-dependent potential drops at the $\mathrm{S}\left(\mathrm{CH}_{2}\right)_{n} \mathrm{X} / /$ electrode interface that modifies the tunnelling barrier shape. Commonly-used tunnelling models neglect localized potential drops and changes in $\varepsilon_{\mathrm{r}}$. Here, we demonstrate experimentally that $\beta \propto 1 / \sqrt{\varepsilon_{r}}$, suggesting highly-polarizable terminal-atoms act as charge traps and highlighting the need for new charge transport models that account for dielectric effects in molecular tunnelling junctions.

\footnotetext{
${ }^{1}$ Department of Chemistry, National University of Singapore, Singapore, Singapore. ${ }^{2}$ Centre for Advanced 2D Materials and Graphene Research Centre, National University of Singapore, Singapore, Singapore. ${ }^{3}$ Department of Physics, Technical University of Munich, Garching, Germany. ${ }^{4}$ Department of Physics, University of Central Florida, Orlando, FL, USA. ${ }^{5}$ Singapore Synchrotron Light Source, National University of Singapore, Singapore, Singapore. ${ }^{6}$ Department of Physics, Bernal Institute, University of Limerick, Limerick, Ireland. ${ }^{7}$ Hybrid Materials for Opto-Electronics Group, Department of Molecules and Materials, MESA+ Institute for Nanotechnology and Center for Brain-Inspired Nano Systems, Faculty of Science and Technology, University of Twente, P.O. Box 217, 7500 AE Enschede, The Netherlands. ${ }_{\text {email: david.egger@tum.de; c.a.nijhuis@utwente.nl }}$
} 
S ignificant effort has been dedicated to study and manipulate tunnelling rates across molecular wires, which serve as model systems to improve our understanding of the mechanisms of charge transport across molecules which, in turn, play a central role in, e.g., biological processes, catalysis, and energy conversion ${ }^{1-4}$. It is well-known that the tunnelling current density $\left(J\right.$ in $\left.\mathrm{A} / \mathrm{cm}^{2}\right)$ decreases exponentially with the length of the molecular wire ( $d$ in $\AA$ ) given by the general tunnelling equation

$$
J=J_{0}(V) \mathrm{e}^{-\beta d}=J_{0}(V) 10^{-\beta d / 2.303},
$$

where $J_{0}$ is a pre-exponential factor and the tunnelling decay coefficient $\left(\beta\right.$ in $\left.\AA^{-1}\right)$ determines how quickly the measured current decays with $d^{2,5-7}$. In this context, unsaturated molecules with conjugated $\pi$-bonds are usually thought of as "molecular conductors" with low values of $\beta\left(0.1-0.4 \AA^{-1}\right)^{2,5,8,9}$ and saturated molecular wires with localized $\sigma$-bonds provide "molecular insulators" with large values of $\beta\left(0.8-1.2 \AA^{-1}\right)^{2,5,7,10,11}$. This rule of thumb stands in sharp contrast with the high tunnelling rates established for various biomolecules ${ }^{12,13}$, molecular wires of oligopeptides $^{14,15}$, and oligosilanes ${ }^{16}$. These all have saturated molecular backbones yet they exhibit low values of $\beta\left(0.1-0.5 \AA^{-1}\right)$, and support long-range tunnelling over remarkably large distances of up to tens of nanometres ${ }^{13,17}$.

So far, it has been challenging to engineer $\beta$ in experiments, and this difficulty is also reflected in various established mechanisms of charge transport across molecular wires. Often, coherent tunnelling is assumed (Eq. 1), where $\beta$ can be related to the tunnelling barrier height $\delta E_{\mathrm{ME}}$ (defined by the offset in energy between the energy of the Fermi level, $E_{\mathrm{F}}$, of the electrode and the energy of the molecular frontier orbital relevant for charge transport), as $\beta \propto \sqrt{\delta E_{\mathrm{ME}}} 2,5,18$. This explains why conjugated molecules, which often have frontier orbitals aligned close to $E_{\mathrm{F}}$, have lower values of $\beta$ than saturated molecules, which have frontier orbitals further from $E_{\mathrm{F}}$. Conversely, in the McConnell superexchange model, charge carriers tunnel via virtual states defined by the repeat units of the molecular wire; here, the tunnelling rate depends on the interaction strength between the repeat units of the molecular bridge $^{2,5,19-21}$. This model has been used to explain low $\beta$ values $\left(0.2-0.5 \AA^{-1}\right)$ measured across tunnel junctions with selfassembled monolayers (SAMs) that have $\sigma$-bond backbones of oligoglycines $^{14}$, oligoprolines ${ }^{22}$, and oligoglycols ${ }^{15}$. Furthermore, for very long molecules (e.g., proteins), a flickering resonance model has been proposed to explain long-range tunnelling and low $\beta$ values $13,23,24$. Finally, different types of hopping models have been proposed to explain low $\beta$ values of, for instance, bacterial nanowires ${ }^{23}, \mathrm{DNA}^{25}$, proteins ${ }^{26}$, and long conjugated molecular wires ${ }^{16,27,28}$; here the value of $\beta$ also depends on the coupling strength between the repeat units, but these models predict a thermally activated component ${ }^{27,28}$. To summarize, all previous models suggest the necessity of tuning the chemical nature of the molecular wire to change the value of $\beta$.

We note that the value of $\beta$ also depends, besides the chemical nature of the molecular backbone $e^{2,5,13-15}$, on the coupling strength between the molecules and electrodes $(\Gamma)$ that is naturally related to $\delta E_{\mathrm{ME}}{ }^{28-30}$. For molecular wires, where $\delta E_{\mathrm{ME}}$ decreases with the number of repeat units due to an increase in conjugation with increasing molecular length, extremely low $\left(<0.1 \AA^{-1}\right)^{27,28,31,32}$ and even negative $\beta$ values have been reported ${ }^{31,33-35}$. Such low $\beta$ values are also a signature of incoherent hopping and these junctions, in particular those containing redox centres, may operate in this hopping regime (also called incoherent tunnelling regime) ${ }^{27,28,33}$. Lambert and co-workers ${ }^{36}$ were able to tune the $\beta$ value between 0.06 and $0.39 \AA^{-1}$ in $\mathrm{Au}-\mathrm{S}\left(\mathrm{CH}_{2}\right)_{n} \mathrm{FG}\left(\mathrm{CH}_{2}\right)_{n} \mathrm{~S}-\mathrm{Au}$ junctions with a functional group $\mathrm{FG}=\alpha$-terthiophene, phenyl, or viologen. They found that changing the anchoring group from dithiol to dithiolmethyl for $\mathrm{FG}=$ phenyl resulted in an increase of the $\beta$ value from 0.14 to $0.50 \AA^{-1}$ from which they concluded that localized states on the Au-S bond are involved in tunnelling along the FG units. In contrast, Frisbie and co-workers ${ }^{37}$ found that $\beta$ values are similar for $\mathrm{Au}-\mathrm{S}\left(\mathrm{CH}_{2}\right)_{n} \mathrm{CH}_{3} / / \mathrm{Au}$ and $\mathrm{Au}-\mathrm{S}\left(\mathrm{CH}_{2}\right)_{n} \mathrm{~S}-\mathrm{Au}$ junctions, implying that localized states on the $\mathrm{Au}-\mathrm{S}$ bond are not important for tuning $\beta$ (but note that they still significantly affect the contact resistance). Frisbie and co-workers ${ }^{38,39}$ suggested that Stark effects are important to consider as they can cancel the potential effects of localized anchoring group-electrode states. Indeed, strong $\mathrm{Au}-\mathrm{S}$ interaction results in severe broadening of the molecular states and therefore the $\mathrm{Au}-\mathrm{S}$ states only occur as weak features in valence band spectra of aliphatic SAMs ${ }^{38}$ (as also observed in the present study), highlighting the need to optimize the $\Gamma$ such that the molecular states remain localized in the molecule. Recently, Chen and co-workers ${ }^{40}$ reported a method using bimetallic electrodes to enhance the conductance of $\mathrm{HO}_{2} \mathrm{C}\left(\mathrm{CH}_{2}\right)_{n} \mathrm{CO}_{2} \mathrm{H}$ single-molecule junctions via the surface $d$-band. They improved the interfacial interactions between molecules and transition metal electrodes, promoting interfacial electron transport. Here, we use junctions of the form $\mathrm{Ag}-\mathrm{S}\left(\mathrm{CH}_{2}\right)_{n} \mathrm{X} / / \mathrm{EGaIn}(n=10,12,14,16$, or 18 , and $\mathrm{X}=\mathrm{H}, \mathrm{F}, \mathrm{Cl}, \mathrm{Br}$, or I) where the weak interaction between the top electrode and the SAM allows us to investigate in detail how the terminal group $\mathrm{X}$ affects the tunnelling rates across the junctions.

So far, the influence of electrostatic effects in molecular tunnelling junctions on $\beta$ has been largely ignored. One way of quantifying trends in the electrostatics of various systems is by studying the static dielectric constant $\left(\varepsilon_{\mathrm{r}}\right)$ of molecular junctions, a macroscopic observable that can be measured via impedance spectroscopy ${ }^{41}$. Previous work focused mainly on $\pi$-conjugated systems and established that in densely packed SAMs, $\varepsilon_{\mathrm{r}}$ hardly changes when the polarizability of the molecules of a SAM, $\alpha$, is tuned due to depolarization effects (e.g., induced dipoles in neighbouring molecules $)^{42-44}$. However, it is not known how $\alpha$ affects the tunnelling behaviour of junctions in which depolarization effects are reduced to a minimum. In addition, the molecular ionization potential directly relates to $\alpha$ and, consequently, changing $\alpha$ affects molecular frontier orbital energies ${ }^{45}$ and the energy level alignment of molecule-electrode interfaces ${ }^{45,46}$, but it is disputed whether an increase in $\alpha$ changes the conductance of the junction ${ }^{45-47}$. Also not currently understood is how $\alpha$ affects the relationship between $\varepsilon_{\mathrm{r}}$ and $\beta$. In principle, polarizable groups screen applied electric fields ${ }^{48}$ or result in an induced dipole and, therefore, also affect the potential drop profile inside junctions ${ }^{45,46}$. Thus polarizable atoms or moieties are expected to have a large effect on the measured tunnelling rates, but so far experimental examples are rare and conflicting ${ }^{45-47}$. For instance, Whitesides and co-workers reported that the charge transport rates in metal-S $\left(\mathrm{CH}_{2}\right)_{n} \mathrm{FG} / / \mathrm{EGaIn}$ junctions with aliphatic SAMs are independent of FG with FG being terminal aromatic groups ${ }^{49}$, polar groups $s^{50}$, ionic and/or hydrogen bonding groups ${ }^{51}$, or halogen atoms ${ }^{47}$, and concluded that changes in terminal group does not affect the charge transport rates. In these studies they used large junction areas of $>1000 \mu \mathrm{m}^{2}$, but we have shown that such large junctions are prone to defects masking molecular effects and that, for EGaInbased methods, stable junctions that are dominated by molecular effects should have an area of $300-500 \mu \mathrm{m}^{2}$ (ref. ${ }^{52}$ ). Indeed, the Whitesides' group could reproduce our results and also found a factor of 600 in the charge transport rates when $\mathrm{X}=\mathrm{H}$ was replaced with $\mathrm{X}=\mathrm{Br}$ when small junctions were used ${ }^{47}$.

Here, we show that the value of $\beta$ of molecular wires with an alkyl chain backbone can be reduced from 0.75 to $0.25 \AA^{-1}$, in effect turning them from insulators into conductors without changing the chemical structure of the backbone of the molecular wire, by introducing one distal polarizable atom at one end of the 
molecular wire of the form $\mathrm{HS}\left(\mathrm{CH}_{2}\right)_{n} \mathrm{X}$. Changing $\mathrm{X}$ from $\mathrm{H}$ to I in the long $\mathrm{S}\left(\mathrm{CH}_{2}\right)_{18} \mathrm{X}$ molecular wire gives a factor of $10^{\approx 5}$ increase in $J$. For $\mathrm{S}\left(\mathrm{CH}_{2}\right)_{10} \mathrm{X}$, the currents change by a factor of $10^{\approx 2}$. As we will discuss below, these observations cannot be explained by changes in the molecule-electrode interfaces, or contact resistances, alone. While we have shown before that the halide group affects the current and $\varepsilon_{\mathrm{r}}$ in $\mathrm{Ag}-\mathrm{S}\left(\mathrm{CH}_{2}\right)_{11} \mathrm{X} / / \mathrm{GaO}_{x} /$ EGaIn junctions ${ }^{45}$, here we demonstrate that the value of $\beta$ can be controlled by changing $\mathrm{X}$ without the need to modify the chemical structure of the molecular backbone. This change in $\beta$ explains why the largest change in current is found for the longest molecules studied in this work. On the basis of experimental and theoretical data, we discuss how introducing this polarizable atom changes the electrostatic potential profile of the tunnelling barrier, the $\varepsilon_{\mathrm{r}}$ of the junction, and the contact resistance (or $\Gamma$ ), which are important to consider when modifying tunnelling efficiency across molecular wires.

\section{Results}

The junctions. Figure 1 shows a schematic illustration of the Ag-S $\left(\mathrm{CH}_{2}\right)_{n} \mathrm{X} / / \mathrm{GaO}_{x} / \mathrm{EGaIn}$ junctions, and indicates how the coupling and energy level alignment (i.e., $\Gamma$ and $\delta \mathrm{E}_{\mathrm{ME}}$ ) change with $\mathrm{X}$ as discussed in detail below. The schematic also includes the equivalent circuit consisting of the contact resistance $\left(R_{\mathrm{C}}\right.$, in $\mathrm{m} \Omega$ $\left.\mathrm{cm}^{2}\right)$ in series with a parallel combination of the SAM resistance $\left(R_{\mathrm{SAM}}\right.$, in $\left.\Omega \mathrm{cm}^{2}\right)$ and the capacitance of the SAM $\left(C_{\mathrm{SAM}}\right.$, in $\mu \mathrm{F} /$ $\mathrm{cm}^{2}$ ) in the junction. The equivalent circuit and the associated physical meaning of each circuit component has been explicitly discussed in our previous work ${ }^{41}$ (and is summarized in Supplementary Section 7). Briefly, the $R_{\mathrm{C}}$ includes the resistances of the contacts of the SAM with the top and bottom electrodes, and the resistance of the electrodes and wires connecting the junction with the electrometers. The SAM itself behaves as a capacitor $\left(C_{\mathrm{SAM}}\right)$ with associated resistance $\left(R_{\mathrm{SAM}}\right)$ as expressed in Eqs. (2) and (3). It highlights that the junctions are essentially parallel plate capacitors in which dielectric behaviour depends on the chemical structure of the junctions, which, as we show below, is also important to explain tunnelling rates. All SAM precursors were synthesized following previously reported methods and characterized with ${ }^{1} \mathrm{H}$ NMR, ${ }^{13} \mathrm{C}$ NMR, and mass spectroscopy (Supplementary Sections 1 and 2). The SAMs were formed on template-stripped Ag electrodes using well-established methods and the junctions were completed with cone-shaped $\mathrm{GaO}_{x} / \mathrm{EGaIn}$ top contacts ${ }^{53}$ (Supplementary Sections 3 and 6). Previously, we have reported that for EGaIn junctions with $\mathrm{S}\left(\mathrm{CH}_{2}\right)_{n} \mathrm{X}$ SAMs (there only $n=11$ was studied) the measured current increased by three orders of magnitude and the value of $\varepsilon_{\mathrm{r}}$ increased by a factor of 4 , when $\mathrm{X}$ was changed along the halogen series from $\mathrm{F}$ to $\mathrm{I}^{45}$. However, the evolution of $J$ and $\varepsilon_{\mathrm{r}}$ with increasing molecular length and the corresponding $\beta$ values for different $\mathrm{X}$ have so far not been studied. Here we address whether this increase in current is caused by changes in $R_{\mathrm{C}}$ or by changes from coherent tunnelling to incoherent process (i.e., $\beta$ ). Changing the value of $n$ for each $\mathrm{X}$ allows us to investigate in detail how and why $\beta$ changes as a function of $\mathrm{X}$ while keeping the nature of the molecule-electrode interfaces and the molecular backbone the same.

Characterization of the SAMs. We characterized the SAMs on Ag with $n=14$ for $\mathrm{X}=\mathrm{H}, \mathrm{F}, \mathrm{Cl}, \mathrm{Br}$, or I, and $n=10,14$, or 18 for $\mathrm{X}=$ $\mathrm{Br}$ with angle-resolved X-ray photoelectron spectroscopy (ARXPS) and molecular dynamics (MD) simulations (for all combinations of $n$ and X) and all results are summarized in Table 1 (see Supplementary Sections 4 and 5 for details). Figure 2a shows a representative snapshot from the MD simulations of $\mathrm{Ag}-\mathrm{S}\left(\mathrm{CH}_{2}\right)_{14} \mathrm{I}$ SAM with computed molecule heights in excellent agreement with film thicknesses $d_{\mathrm{SAM}}$ measured by XPS (Fig. $2 \mathrm{~b}, \mathrm{c}$ and Table 1 ) indicating that the $\mathrm{S}\left(\mathrm{CH}_{2}\right)_{14} \mathrm{I}$ precursor readily forms dense layers with all molecules in a fully-upright position. We determined the relative values of surface coverage $\left(\Psi_{\mathrm{SAM}}\right)$ with XPS, which confirms that all SAMs have indistinguishable packing densities (Fig. 2b, c) within experimental error. The value of $d_{\mathrm{SAM}, \mathrm{MD}}$ increases by about $1.4 \AA$ overall on increasing van der Waals radius of $\mathrm{X}$ from $\mathrm{H}$ to I (Supplementary Table 1), but this small increase falls within the experimental error of $d_{\text {SAM,XPS }}$ (Fig. 2b). Figure $2 \mathrm{c}$ shows that for $\mathrm{X}=\mathrm{Br}, d_{\mathrm{SAM}, \mathrm{XPS}}$ increases linearly with $n$ with a slope of $1.5 \pm 0.1 \AA$ per carbon (solid blue line, error represents standard error from linear fit), which is in close agreement with the MD value of $1.3 \pm 0.1$ A per carbon (dashed blue line; see Supplementary Fig. 11 for $d_{\mathrm{SAM}, \mathrm{MD}}$ values of all the SAMs). Figure $2 \mathrm{~d}$ shows the packing energies per molecule $\left(E_{\mathrm{mol}, \mathrm{MD}}\right.$, in $\mathrm{eV})$ and per methylene $\mathrm{CH}_{2}$ unit $\left(E_{\text {meth,MD }}\right.$, in $\left.\mathrm{meV}\right)$ extracted from the MD calculations. The values of $E_{\text {mol, MD }}$ and $E_{\text {meth }, M D}$ improve slightly as $\mathrm{X}$ shifts from $\mathrm{H}(-1.8 \pm 0.1 \mathrm{eV}$ per molecule) to $\operatorname{Br}(-2.4 \pm 0.2 \mathrm{eV}$ per molecule), which is due to the increasing intermolecular van der Waals interaction. For SAMs with $\mathrm{X}=\mathrm{I}$, the
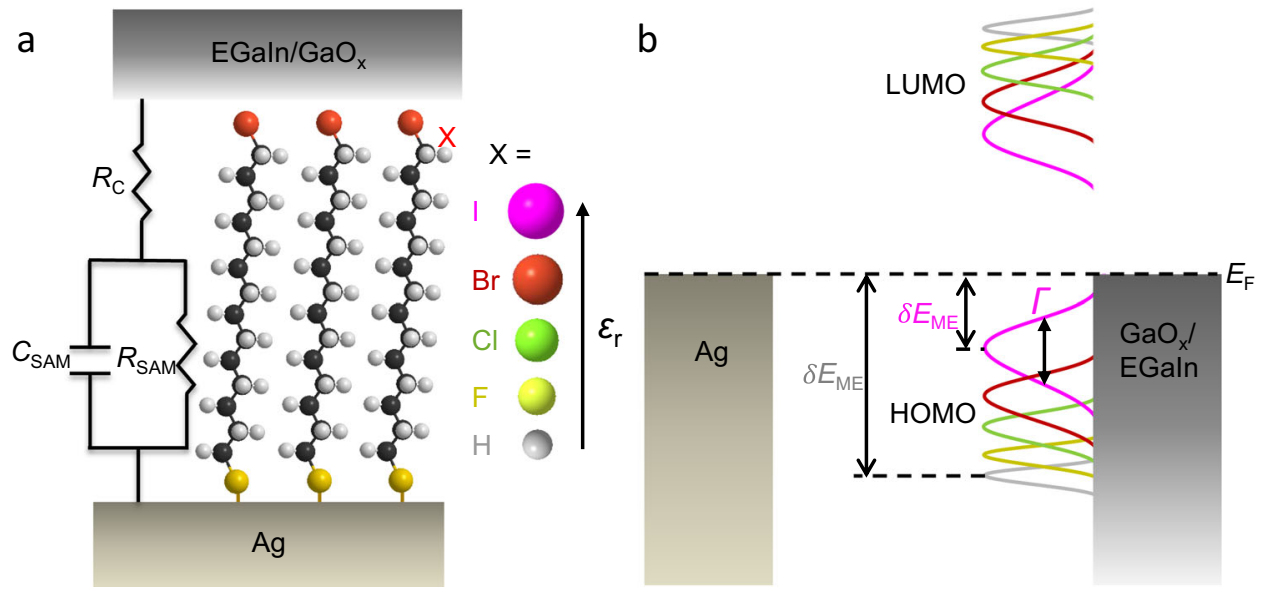

Fig. 1 The junctions, equivalent circuit, and energy level diagram. a Schematic illustration of the $\mathrm{Ag}-\mathrm{S}\left(\mathrm{CH}_{2}\right)_{n} \mathrm{X} / / \mathrm{GaO}_{x} / \mathrm{EGaln}$ junction (shown for $n=14$, EGaln is for eutectic alloy of Gallium and Indium, "-" represents covalent bond, "//" represents non-covalent contact, "/" means the interface between $\mathrm{GaO}_{x}$ and $\mathrm{EGaln)}$ together with the equivalent circuit diagram. In this work we investigated junctions with $n=10,12,14,16$, or 18, and $\mathrm{X}=\mathrm{H}, \mathrm{F}, \mathrm{Cl}, \mathrm{Br}$, or I. $\mathbf{b}$ Energy level diagram of the junction showing how the coupling strength between molecules and electrodes $(\Gamma)$ and tunnelling barrier height $\left(\delta E_{\mathrm{ME}}\right)$ change with X. 
Table 1 Summary of properties of the $\mathrm{Ag}-\mathrm{S}\left(\mathrm{CH}_{2}\right)_{n} \mathrm{X}$ SAMs.

\begin{tabular}{|c|c|c|c|c|c|c|c|c|}
\hline$X$ and $n$ & $\Psi_{\text {SAM }, X P S}\left(\mathrm{nmol} / \mathrm{cm}^{2}\right)^{\mathrm{a}}$ & $d_{\text {SAM }, X P S}(\AA)$ & $d_{S A M, M D}(\AA)$ & $E_{\text {mol,MD }}(\mathrm{eV})$ & $\Phi_{\text {SECO }}(\mathrm{eV})^{\mathrm{b}}$ & $\Phi_{\mathrm{DFT}}(\mathrm{eV})$ & $\varepsilon_{\mathrm{r}}$ & $\varepsilon_{\text {DFT-VdW }}$ \\
\hline$n=14, X=F$ & 1.0 & 21 & $21.1 \pm 0.3$ & $-2.0 \pm 0.1$ & 4.43 & 5.44 & $2.5 \pm 0.6$ & 2.1 \\
\hline$n=14, X=C l$ & 0.86 & 21 & $21.5 \pm 0.3$ & $-2.2 \pm 0.2$ & 5.02 & 5.25 & $3.0 \pm 0.2$ & 2.2 \\
\hline$n=14, X=1$ & 1.2 & 21 & $21.8 \pm 0.3$ & $-2.2 \pm 0.1$ & 4.68 & 4.96 & $8.9 \pm 1.6$ & 2.4 \\
\hline$n=10, X=B r$ & 1.0 & 15 & $16.2 \pm 0.4$ & $-1.7 \pm 0.1$ & 4.62 & - & $4.4 \pm 0.4$ & - \\
\hline$n=18, X=B r$ & 1.1 & 29 & $26.8 \pm 0.3$ & $-3.0 \pm 0.2$ & 4.66 & - & $4.6 \pm 0.2$ & - \\
\hline
\end{tabular}

a
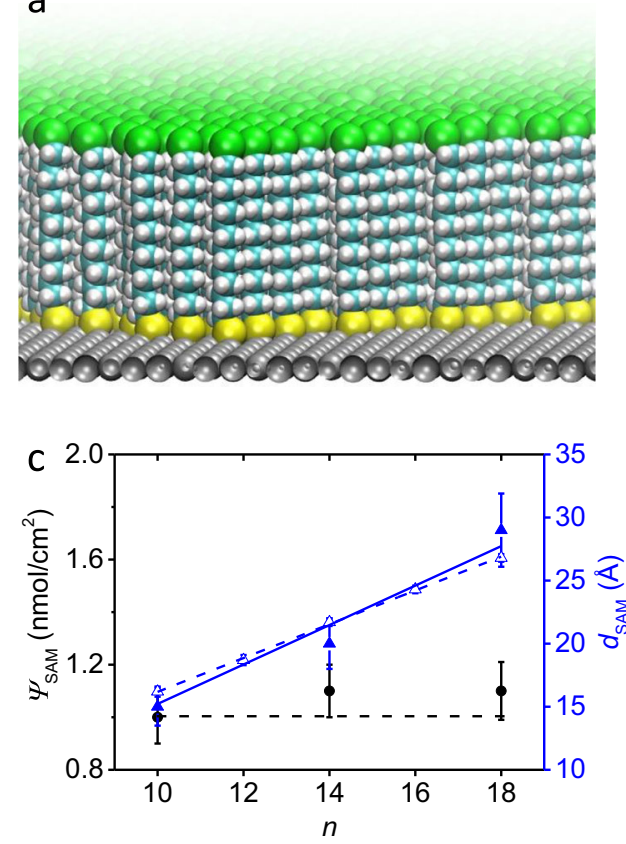

b
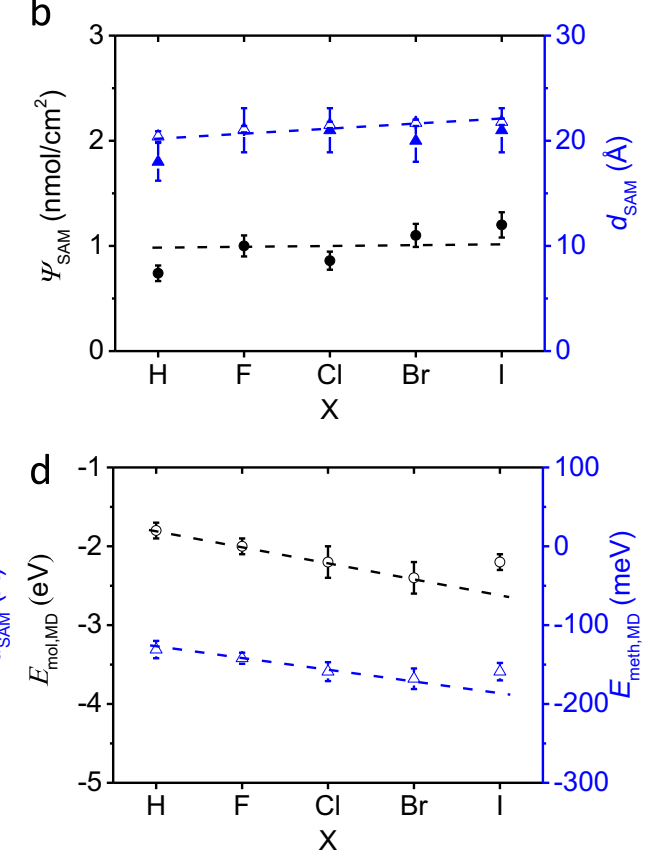

Fig. 2 Characterization of the self-assembled monolayers (SAMs). a Representative slice-through of a large-area $\mathrm{Ag}-\mathrm{S}\left(\mathrm{CH}_{2}\right)_{14} \mid \mathrm{SAM}$ structure calculated by molecular dynamics (MD) computer simulations. b Surface coverage $\left(\Psi_{\text {SAM }}\right)$ of $\mathrm{Ag}-\mathrm{S}\left(\mathrm{CH}_{2}\right)_{14} \mathrm{X}$ SAMs as a function of $\mathrm{X}$ determined with angle-resolved $X$-ray photoelectron spectroscopy (ARXPS, filled circles) and thickness of SAM ( $d_{S A M}$ ) determined with ARXPS (filled triangles) and MD (empty triangles). c $\Psi_{\text {SAM }}$ of Ag-S $\left(\mathrm{CH}_{2}\right)_{n} \mathrm{Br}$ SAMs as a function of $n$ determined with ARXPS (filled circles) and $d_{\text {SAM }}$ determined with ARXPS (filled triangles) and MD (open triangles). The solid and dashed blue lines are linear fits to the experimental and MD data with $R^{2}$ of 0.94 and 0.99 , respectively. The horizontal dashed line in panels $\mathbf{b}$ and $\mathbf{c}$ indicates the $\Psi_{\text {SAM }}$ used in the MD calculations. $\mathbf{d}$ Computed MD packing energy per molecule $E_{\text {mol,MD }}$ and per methylene $-\mathrm{CH}_{2}-$ unit $E_{\text {meth,MD }}$ of $\mathrm{Ag}-\mathrm{S}\left(\mathrm{CH}_{2}\right)_{14} \mathrm{XSAMs}$ as a function of X. Dashed lines are guides to the eye. The errors on the XPS data represent instrumental and fitting errors of $10 \%$ in total (see Section S4). The error bars in the MD data represent the standard deviations in the time- and molecule-averages calculated across 500 snapshots taken during the final $50 \mathrm{~ns}$ of $100 \mathrm{~ns}$ of room temperature MD of 128 -molecule $\mathrm{Ag}-\mathrm{S}\left(\mathrm{CH}_{2}\right)_{14} \mathrm{X}$ SAMs with the average experimental coverage of $1 \mathrm{nmol} / \mathrm{cm}^{2}$ on $\mathrm{Ag}(111)$.

packing energies weaken slightly due to small competing effects caused by mild steric repulsion between the large I headgroups. These observations confirm that the halogen functionality does not significantly disrupt the supramolecular structure of the SAM. Finally, we determined the energy level alignment of the SAMs on Ag using ultra-violet photoemission spectroscopy in Supplementary Section 4 and Supplementary Fig. 10, which we used to validate our densityfunctional theory (DFT) calculations as discussed in more detail below (Table 1).

Electrical characterization of the junctions. To study how the halogen functionality affects the tunnelling rates across the SAMs, we measured the electrical characteristics of the junctions as a function of $\mathrm{X}$ and $n$ using $J(V)$ measurements and impedance spectroscopy. The SAMs were contacted with cone-shaped $\mathrm{GaO}_{x}$ l EGaIn electrodes following a previously reported method ${ }^{53}$. To minimize leakage currents and to ensure that molecular effects dominate the junction characteristics, we used junctions with a small contact area of $\sim 350 \mu \mathrm{m}^{2}$, as large junctions suffer from leakage currents across defective sites ${ }^{52}$. We recorded statistically large numbers of $J(V)$ curves to determine the Gaussian log-average $J(V)$ curves, $<\log _{10}|J|>{ }_{\mathrm{G}}$, and associated Gaussian log-standard deviations $\left(\sigma_{\log , \mathrm{G}}\right)$ which are plotted in Fig. 3a for junctions with $\mathrm{X}=\mathrm{F}$, and in Fig. $3 \mathrm{~b}$ for junctions with $\mathrm{X}=\mathrm{I}$, for $n=10-18$ (all Gaussian log-average $J(V)$ curves and histograms of the $\log _{10}|J|$ at $\pm 0.5 \mathrm{~V}$ are given in Supplementary Section 6). Clearly, the tunnelling rates are more attenuated for $\mathrm{X}=\mathrm{F}$ than for $\mathrm{X}=\mathrm{I}$. Figure $3 \mathrm{c}$ 

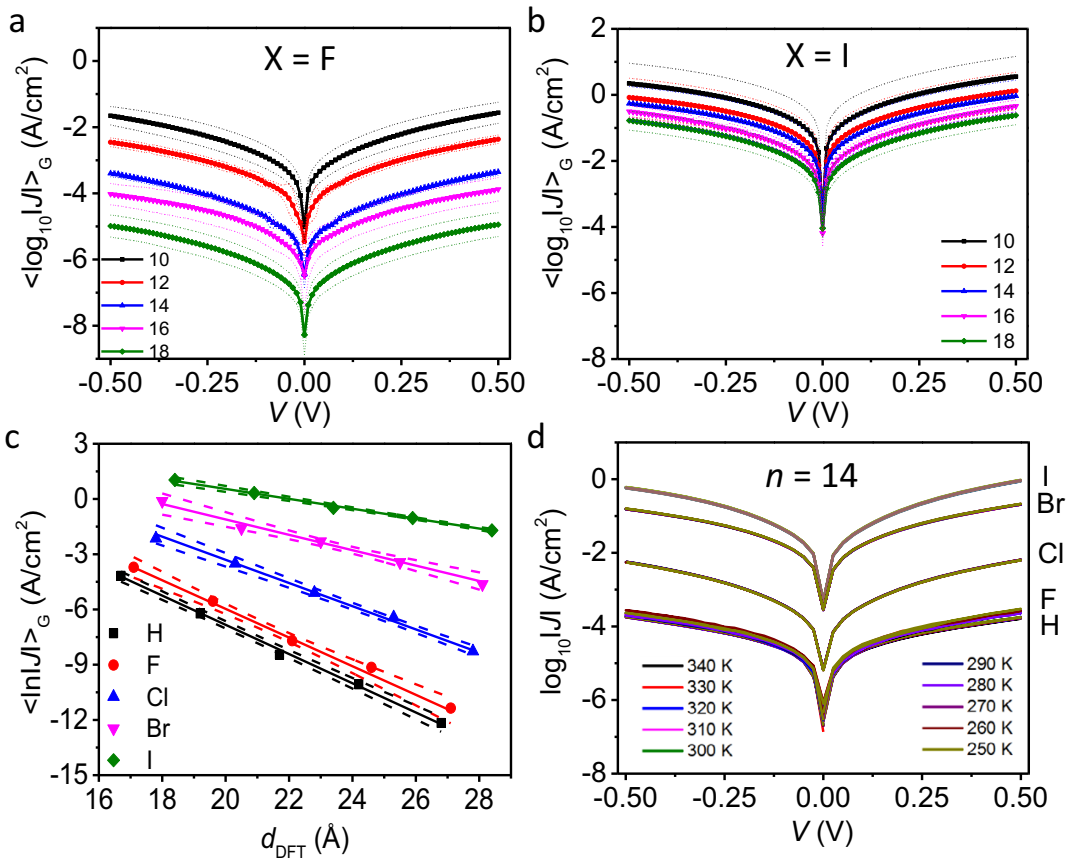

Fig. 3 Electrical characterization of junctions. Gaussian log-average values of the current densities $\left\langle\log _{10}|J|\right\rangle_{G}$ vs. applied bias $V$ obtained from Ag-S $\left(\mathrm{CH}_{2}\right)_{n} \mathrm{X} / / \mathrm{GaO}_{x} / \mathrm{EGaln}$ junctions with $\mathrm{X}=\mathrm{F}$ (a) or I (b) and $n=10$ (solid black line), 12 (solid red line), 14 (solid blue line), 16 (solid pink line), and 18 (solid green line). The dashed-line error bars represent the Gaussian log-standard deviation, $\sigma_{\log , G}$. c Decay plots of $\left\langle\log _{10}|J|>_{\mathrm{G}}\right.$ at $-0.5 \mathrm{~V}$ against $d_{\mathrm{SAM}, \mathrm{MD}}$ with $\mathrm{X}=\mathrm{H}$ (black square), $\mathrm{F}$ (red circle), $\mathrm{Cl}$ (blue triangle), $\mathrm{Br}$ (pink inverted triangle), or I (green diamond). The solid lines are fits to Eq. (1). The dashed lines represent the $95 \%$ confidence bands. $d$ Plots of $\log _{10}|J|$ vs. $V$ as a function of $T(T=250-340 \mathrm{~K})$ recorded from $\mathrm{Ag}-\mathrm{S}(\mathrm{CH})_{14} \mathrm{X} / / \mathrm{GaO} \times / \mathrm{EGaln}$ junctions

shows the decay of $\left\langle\log _{10}|J|>_{\mathrm{G}}\right.$ at $-0.5 \mathrm{~V}$ as a function of $d_{\mathrm{SAM}, \mathrm{MD}}$ (Supplementary Section 5 and Supplementary Table 1) for all X. The solid lines are fits to Eq. (1) from which we determined the values of $\beta$ which are listed in Supplementary Table 7. Supplementary Figure 18 shows the plot of $\beta$ vs. X. Interestingly, the value of $\beta$ steadily decreases from $0.75 \pm 0.01 \AA^{-1}$ for $\mathrm{X}=\mathrm{H}-\mathrm{a}$ typical value for tunnelling along alkyl chains-to $0.25 \pm 0.01 \AA^{-1}$ for $\mathrm{X}=\mathrm{I}$ which is a typical value for tunnelling along $\pi$-conjugated molecules (the error in $\beta$ represents the standard error of the fit to Eq. 1). We measured the $J(V)$ characteristics as a function of temperature, $T$ in $\mathrm{K}$, of $\mathrm{Ag}-\mathrm{S}\left(\mathrm{CH}_{2}\right)_{14} \mathrm{X} / / \mathrm{GaO}_{x} / \mathrm{EGaIn}$ junctions for all $\mathrm{X}$ using top electrode of EGaIn confined in a microfluidic network in polydimethylsiloxane following a previous reported method ${ }^{41}$ (see Supplementary Section 6 for details). Figure 3d shows that the tunnelling rates are independent of $T$ in the range of $T$ from 250 to $340 \mathrm{~K}$, which is consistent with coherent off-resonant tunnelling 54 .

Dielectric constant of the junctions. To characterize the dielectric response of the junctions, we conducted impedance spectroscopy using a sinusoidal voltage perturbation with an amplitude of $30 \mathrm{mV}$ around $0 \mathrm{~V}$ in the frequency range of $100 \mathrm{~Hz}$ to $1.00 \mathrm{MHz}$ and the data were fitted to the equivalent circuit shown in Fig. 1a following a previously reported method ${ }^{41}$ (see Supplementary Section 7 for details). Supplementary Figure 23 shows the Bode, Nyquist, and the corresponding phase angle (5) vs. frequency $(f)$ plots along with the fits to the equivalent circuit (Supplementary Tables 8-10 list all fitting results). Figure $4 \mathrm{a}$ shows that $R_{\mathrm{C}}$ decreases by a factor of 5 when $\mathrm{X}$ is changed from $\mathrm{H}$ or $\mathrm{F}$ to I while $R_{\mathrm{C}}$ is independent of $n$ (Fig. $4 \mathrm{~b}$ ). This change in $R_{\mathrm{C}}$ indicates that $\Gamma$ substantially increases as a function of $\mathrm{X}$. This increase in $\Gamma$ can be rationalized by the increase in polarizability $\alpha$ and associated induced dipoles as a function of $\mathrm{X}$ resulting in an increase in the van der Waals interaction strength between the SAM and the top contact ${ }^{45}$. Supplementary Table 9 shows the decrease of $R_{\mathrm{SAM}}$ with $\mathrm{X}$ which is mainly caused by lowering of $\delta E_{\mathrm{ME}}$ and increase of $\Gamma$ (see the "DFT Calculations" section). Frisbie and co-workers ${ }^{9,38}$ have shown that a decrease in $R_{\mathrm{C}}$ by increasing the work function of the bare metal electrodes increases the conductivity of molecular junctions (with $\mathrm{H}$ or $\mathrm{S}$ terminal atoms), which was mainly driven by a large increase in $\Gamma$, with changes in $\delta E_{\mathrm{ME}}$ and $\beta$ playing only a minor role.

To confirm the consistency between the $J(V)$ and impedance measurements, we determined the value of $\beta$ from the impedance measurements for junctions with $\mathrm{X}=\mathrm{Br}$. The value of $R_{\mathrm{SAM}}$ increases exponentially with $n$ (Eq. 2)

$$
R_{\mathrm{SAM}}=R_{\mathrm{SAM}, 0}(V) \mathrm{e}^{\beta d_{\mathrm{SAM}, \mathrm{MD}}}=R_{\mathrm{SAM}, 0}(V) 10^{\beta d_{\mathrm{SAM}, \mathrm{MD}} / 2.303}
$$

where $R_{\mathrm{SAM}, 0}$ is a pre-exponential factor. Figure $4 \mathrm{c}$ shows the plot of $\log _{10} R_{\text {SAM }}$ vs. $n$ along with a fit to Eq. (2) from which we extracted the values of $\beta=0.41 \pm 0.03 \AA^{-1}$ and $\log _{10} R_{\mathrm{SAM}, 0}=$ $-2.0 \pm 0.2 \Omega / \mathrm{cm}^{2}$ (or $R_{\mathrm{SAM}, 0}=1.0 \times 10^{-2} \Omega / \mathrm{cm}^{2}$ ). The value of $R_{\mathrm{SAM}, 0}$ is essentially equivalent to $J_{0}$ (defined in Eq. 1 ) derived from a current decay plot at $30 \mathrm{mV}$ (since the sinusoidal perturbation used in the impedance measurements was $30 \mathrm{mV}$ ). The value of $J_{0}$ at $30 \mathrm{mV}$ is $5.1 \pm 2.0 \mathrm{~A} / \mathrm{cm}^{2}$ and the $\beta=0.46 \pm 0.03 \AA^{-1}$ (Supplementary Fig. 17). $R_{\mathrm{SAM}, 0} \approx V / J_{0}=0.59 \times 10^{-2} \Omega / \mathrm{cm}^{2}$, which is within a factor of 2 of the value measured with impedance spectroscopy $\left(R_{\mathrm{SAM}, 0}=1.0 \times 10^{-2} \Omega / \mathrm{cm}^{2}\right)$. The contribution of $R_{\mathrm{C}}$ is minor since $R_{\mathrm{C}}$ is a parallel circuit element, but it is included in $J_{0}$. The $\beta$ and $R_{\mathrm{SAM}, 0}$ values are, within error, the same as the values determined with the $J(V)$ measurements.

To gain further insight into the dielectric properties of the junctions, we used the parallel plate capacitor equation (Eq. 3 ) to determine $\varepsilon_{\mathrm{r}}$ as a function of $\mathrm{X}$ and $n$

$$
C_{\mathrm{SAM}}=\varepsilon_{0} \varepsilon_{r} \frac{A_{\text {geo }}}{d_{\mathrm{SAM}, \mathrm{MD}}}
$$

wherein $\varepsilon_{0}$ is the vacuum permittivity and $A_{\text {geo }}$ is the geometrical 

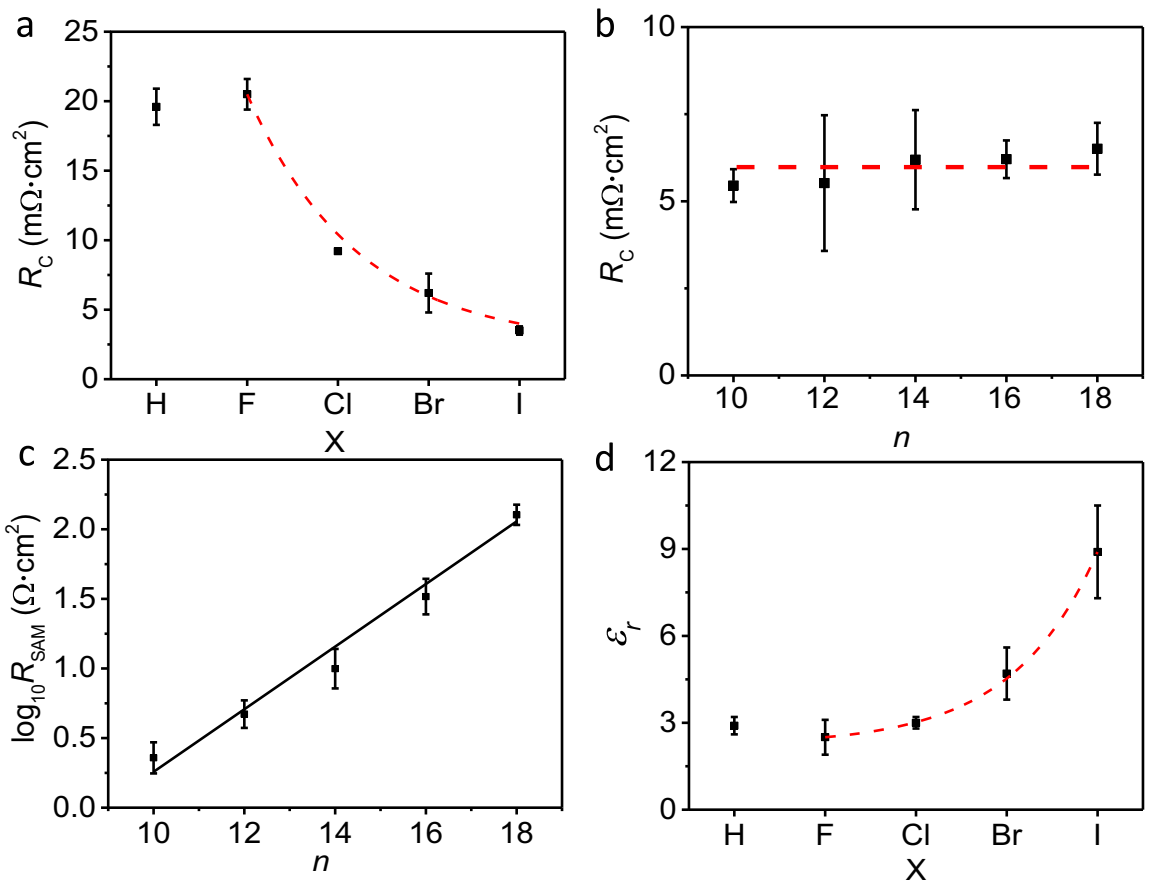

Fig. 4 Characterization of junctions with impedance spectroscopy. a Contact resistance $R_{\mathrm{C}}$ vs. $\mathrm{X}$ for $\mathrm{Ag}-\mathrm{S}\left(\mathrm{CH}_{2}\right)_{14} \mathrm{X} / / \mathrm{GaO}_{x} / \mathrm{EGaln}$ junctions at $\mathrm{DC}$ of $\mathrm{O} \mathrm{V}$ and sinusoidal perturbation of $30 \mathrm{mV}$. Log-resistance of $\mathrm{SAM}, \log _{10} R_{\mathrm{SAM}}(\mathbf{b})$ and $R_{\mathrm{C}}(\mathbf{c})$ vs. $n$ for $\mathrm{Ag}-\mathrm{S}\left(\mathrm{CH}_{2}\right)_{n} \mathrm{Br} / / \mathrm{GaO}_{x} / \mathrm{EGaln}$ junctions. The solid black line represents a fit to Eq. (2). d Corresponding dielectric constant $\varepsilon_{r}$ vs. $X$ for $A g-S\left(C_{2}\right)_{14} X / / G_{a O} / E G a l n$ junctions. The error bars are the standard deviations of three independent measurements. Dashed lines are visual guides.

area of the junction. Figure $4 \mathrm{~d}$ shows that $\varepsilon_{\mathrm{r}}$ increases by a factor of 3 when changing $\mathrm{X}$ from $\mathrm{H}$ or $\mathrm{F}$ to $\mathrm{I}$, yet $\varepsilon_{\mathrm{r}}$ is independent of $n$ (Supplementary Tables 9-10). Although this factor 3 increase in $\varepsilon_{\mathrm{r}}$ is expected for bulk systems which can be described via the Clausius-Mosotti relation ${ }^{55}$, this observation cannot be explained as an intrinsic electrostatic property of the molecular wires, as we will show and discuss below. Moreover, even though we can quantify the contributions of different circuit components from impedance spectroscopy, how these components are influenced by each other are not directly revealed. Therefore, we referred to DFT and Landauer modelling for further explanations.

DFT calculations. To provide further microscopic insight into the electrostatic properties and electronic structure of the molecular wires, we performed first-principles calculations based on DFT using the VASP code ${ }^{56}$ and a $3 \times 2 \sqrt{ } 3$ Ag surface unit cell containing four molecules arranged in a herringbone pattern (see Supplementary Section 8 for full details).

Figure 5a shows that the shape of the potential energy towards the tail of the alkyl chain strongly depends on the functionalization at the $\mathrm{X}$-site. Specifically, the vacuum level changes with $\mathrm{X}$ functionalization owing to the polarity of the $\mathrm{C}-\mathrm{X}$ bond, which translates into a change of the Ag work function, $\Phi$, as expected for SAMs with different tail groups $45,46,57,58$. Comparing the DFT-calculated $\Phi$ to the experimental $\Phi$, it can be seen that the agreement is good for all terminations except $\mathrm{X}=\mathrm{F}$ (Fig. 5b). We tentatively ascribe the quantitative deviations to the often observed overestimation of polar effects in periodic DFT calculations of metal-SAM interfaces due to the assumption of perfect molecular order and periodicity ${ }^{59}$, while practical systems have defects (e.g., step edged, grain boundaries, or phase domains) and are dynamic in nature 52,60 .

Figure $5 \mathrm{c}$ reports the density of states (DOS) projected onto the molecular part of $\mathrm{Ag}-\mathrm{S}\left(\mathrm{CH}_{2}\right)_{14} \mathrm{X}$. All systems show a feature at $\sim 1.5 \mathrm{eV}$ (marked by ${ }^{*}$ in Fig. $5 \mathrm{c}$ ) that is due to Ag-S hybridization
(Supplementary Section 8). Interestingly, we find that lower lying occupied states as well as the lowest unoccupied state strongly shift in energy (on the order of $1-2 \mathrm{eV}$ ) with varying $\mathrm{X}$ (see arrows in Fig. 5c). These energy shifts clearly correlate with $\mathrm{X}$ functionalization and increase in magnitude along the halogen series, so that the $\mathrm{X}=\mathrm{I}$ SAM shows pronounced new features close to the band edges when compared to the $\mathrm{X}=\mathrm{H}$ or $\mathrm{X}=\mathrm{F}$ SAM. Figure $5 \mathrm{~d}$ shows the DOS projected onto just the $\mathrm{X}$-site in $\mathrm{Ag}-\mathrm{S}\left(\mathrm{CH}_{2}\right)_{14} \mathrm{X}$, which confirms that these new occupied and unoccupied states are due to the halogen functionalization. For both the occupied and unoccupied parts of the DOS, these halogen-derived states do partially overlap in energy with other features but are localized primarily at the tail of the SAM (Supplementary Section 8). The $\mathrm{X}$ groups are not redox-active, and even for $\mathrm{X}=\mathrm{I}$ the $\mathrm{HOMO}$ is still $\sim 1.7 \mathrm{eV}$ below $E_{\mathrm{F}}$ (Fig. $5 \mathrm{c}, \mathrm{d}$ ). Hence, the HOMO cannot enter the applied bias window of $\pm 0.5 \mathrm{~V}$ (note the molecules with $n=10$ tend to break down at higher voltages $)^{61}$.

Finally, we determined $\varepsilon_{\mathrm{r}}$ as a function of $\mathrm{X}$ for the freestanding and hydrogen-terminated $\mathrm{HS}\left(\mathrm{CH}_{2}\right)_{14} \mathrm{X}$ SAMs using previously reported protocols ${ }^{44,62}$ (see Table 1 and Supplementary Section 8). Hereby, $\varepsilon_{\mathrm{r}}$ is calculated from the change in the dipole moment induced by an applied static electric field, with the atomic positions fixed at their equilibrium positions. Thus, the $\varepsilon_{\mathrm{r}}$ obtained in such a manner represents the instantaneous response of the electronic charge density to a static electric field. In contrast to the above-discussed experimental results, we find that $\varepsilon_{\mathrm{r}}$ hardly changes with $\mathrm{X}$ functionalization in our DFT calculations. This result is expected from purely electrostatic reasoning and fully in line with previous work by various groups ${ }^{42-44}$. Briefly, in these studies it has been shown from electrostatic and DFT calculations that varying the molecular polarizability of the SAM-forming molecules does not result in significant changes of $\varepsilon_{\mathrm{r}}$ in the densely packed conjugated SAMs due to depolarization effects arising from the neighbouring molecular dipoles in the SAM ${ }^{42-44}$. Therefore, the calculations show that tuning the molecular 

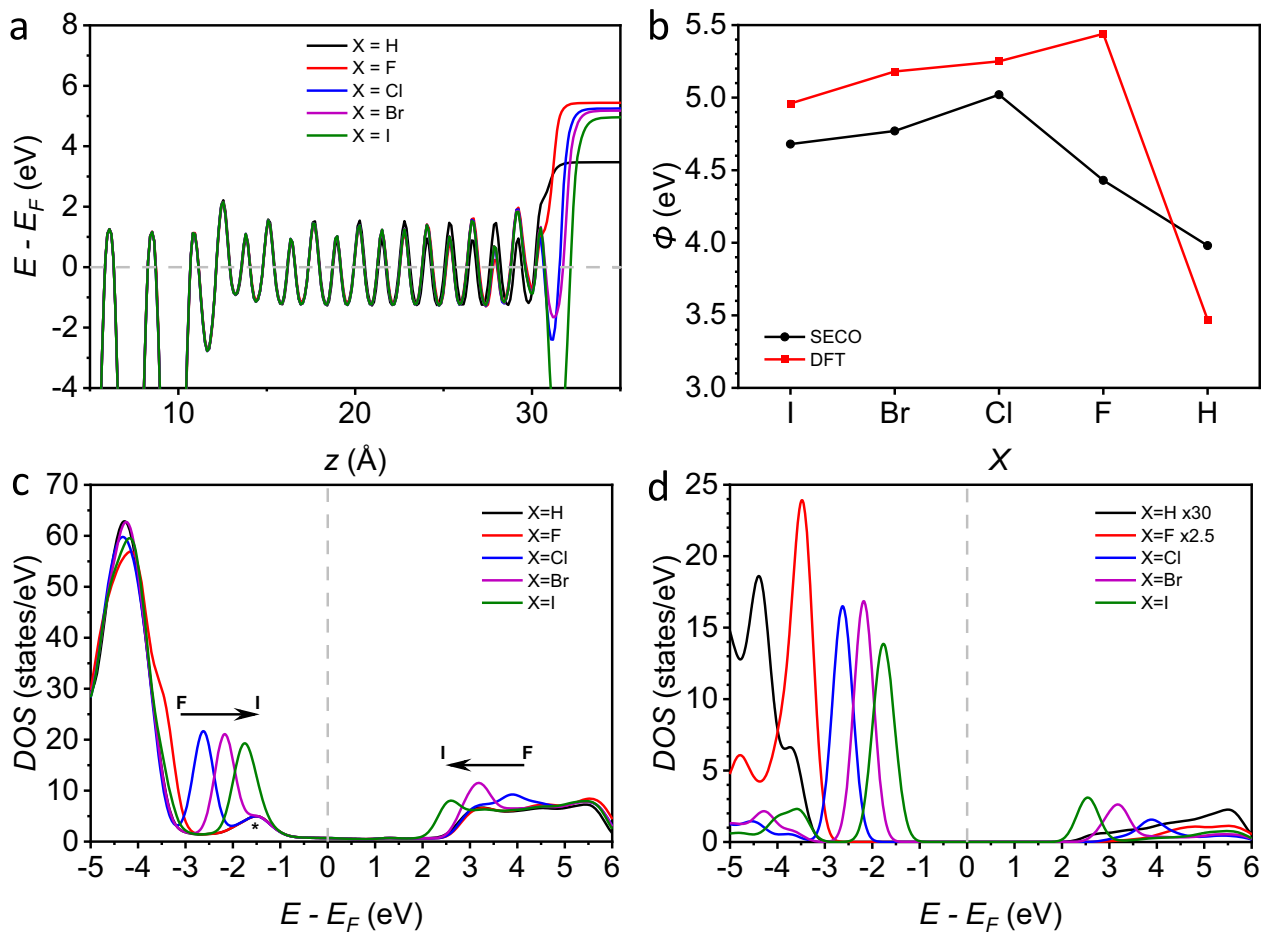

Fig. 5 Density-functional theory (DFT) calculations. a DFT-calculated plane-averaged electrostatic potential of $\mathrm{Ag}(111)-\mathrm{S}(\mathrm{CH})_{14} \mathrm{X}$ where $\mathrm{X}=\mathrm{H}$, $\mathrm{F}, \mathrm{Cl}, \mathrm{Br}$, or I, along the surface-normal coordinate. $\mathbf{b}$ Work function $(\Phi)$ of the SAMs, calculated from DFT (red squares) and measured experimentally (black dots). Density of states (DOS) projected onto the molecular backbone (c) and onto the $\mathrm{X}$-site $(\mathbf{d})$ of $\mathrm{Ag}(111)-\mathrm{S}\left(\mathrm{CH}_{2}\right){ }_{14} \mathrm{X}$. Note that $\mathrm{X}$ contributes very little to the band edges for $\mathrm{X}=\mathrm{H}, \mathrm{F}$.

polarizability by changing $\mathrm{X}$ does not strongly impact the calculated $\varepsilon_{\mathrm{r}}$ of the $\mathrm{HS}\left(\mathrm{CH}_{2}\right)_{14} \mathrm{X}$ SAMs.

As pointed out by Natan et $a .^{43}$, a competition between suppression of in-plane polarization and enhancement of out-ofplane polarization occurs in SAMs. The suppression dominates for densely-packed SAMs and, thus, the substituent $\mathrm{X}$ should not affect the calculated $\varepsilon_{\mathrm{r}}$ of the SAM in sharp contrast to our herin reported experimental findings. Our calculations as well as previous theoretical studies, however, only probe the intrinsic dielectric properties of the isolated highly organized SAM without contacts. The interaction between the SAM and the top electrode that is naturally present in the experimental determination of $\varepsilon_{\mathrm{r}}$ could affect the dielectric behaviour of the junction considerably, which would be consistent with the experimentally recorded trends for the $R_{\mathrm{C}}$ shown in Fig. 4a. Given the high electric fields on the order of $\mathrm{GV} / \mathrm{m}$ and the polarizable nature of $\mathrm{X}$, the substituents may be partially charged during charge transport (especially iodines are well-known to readily accommodate electrons) ${ }^{63,64}$. We note that previously reported DFT calculations of $\varepsilon_{\mathrm{r}}$ of the $\mathrm{HS}\left(\mathrm{CH}_{2}\right)_{11} \mathrm{X} \mathrm{SAMs}^{45}$ were incorrect due to simulation artefacts of uncompensated dipoles in the unit cell, which created a spurious correlation with experimentally measured $\varepsilon_{\mathrm{r}}$ values.

Single-level Landauer model. In the following, we discuss our results in the context of commonly used models to interpret charge transport through the $\mathrm{S}\left(\mathrm{CH}_{2}\right)_{n} \mathrm{X}$ molecular junctions. The single-level Landauer model is frequently used to model the current flowing across molecular tunnel junctions ${ }^{65}$. Here we modelled the current using the following expression:

$$
I=\frac{N q}{h} \iint_{-\infty}^{\infty} \mathrm{d} E \mathrm{~d} E^{\prime} D_{E^{\prime}}(E) G_{\delta E_{\mathrm{ME}}}\left(E^{\prime}\right) \frac{\gamma_{\mathrm{L}} \gamma_{\mathrm{R}}}{\gamma_{\mathrm{L}}+\gamma_{\mathrm{R}}}\left[f_{\mathrm{L}}(E)-f_{\mathrm{R}}(E)\right]
$$

where $\gamma_{\mathrm{L}}$ and $\gamma_{\mathrm{R}}$ are the tunnelling rates between the molecule and the left and right electrodes (respectively), $D_{E^{\prime}}(E)$ is the electronic density of states of the molecular level having the shape of Lorentzian and is given by

$$
D_{E^{\prime}}(E)=\frac{\frac{\gamma}{2 \pi}}{\left(E-\left(E^{\prime}+\left(\eta-\frac{1}{2}\right) \times V\right)^{2}+\left(\frac{y}{2}\right)^{2}\right.}
$$

centred at energy $E^{\prime}+\left(\eta-\frac{1}{2}\right) V$, where $\eta=V_{\mathrm{R}} /\left(V_{\mathrm{L}}+V_{\mathrm{R}}\right)$ is the voltage division parameter accounting for the capacitive coupling with the left and right electrodes, and with a level width $\gamma=\gamma_{\mathrm{L}}+\gamma_{\mathrm{R}}$. The $f_{\mathrm{L}}(E)$ and $f_{\mathrm{R}}(E)$ are the Fermi functions representing the electronic occupation of the left and right electrodes, respectively, which are given by ${ }^{65}$

$$
f_{\mathrm{L}, \mathrm{R}}(E)=\frac{1}{1+\exp \left[\frac{E \pm \frac{V}{2}}{K_{\mathrm{B}} T}\right]}
$$

Equations 4-6 provide the model to which we fitted the experimental data. In addition, we attached a Gaussian to the model with the inherent dispersion $(\sigma)$ of the molecular level energy $\left(\delta E_{\mathrm{ME}}\right.$ ) in an ensemble of molecules (rather than a singlemolecule junction), as given by the following expression:

$$
G_{\delta E_{\mathrm{ME}}}\left(E^{\prime}\right)=A \exp \left(\frac{\left(E^{\prime}-\delta E_{\mathrm{ME}}\right)^{2}}{2 \sigma^{2}}\right)
$$

We accounted for the behaviour of a group of molecules by setting the number of such molecules fixed at $N=150$. As obviously seen in the above model, the current is directly dependent on $\gamma_{\mathrm{L}} \times \gamma_{\mathrm{R}}$. There is a trade-off between the Gaussian and the density of states which is in the shape of a Lorentzian centred at the energy level $\delta E_{\mathrm{ME}}$. All the molecules appeared to be symmetric and what accounts for the difference in conductance is the terminal atom on the molecular unit. In this case, the ligands were $\mathrm{X}=\mathrm{H}, \mathrm{F}, \mathrm{Cl}, \mathrm{Br}$, or I. Therefore five different set 

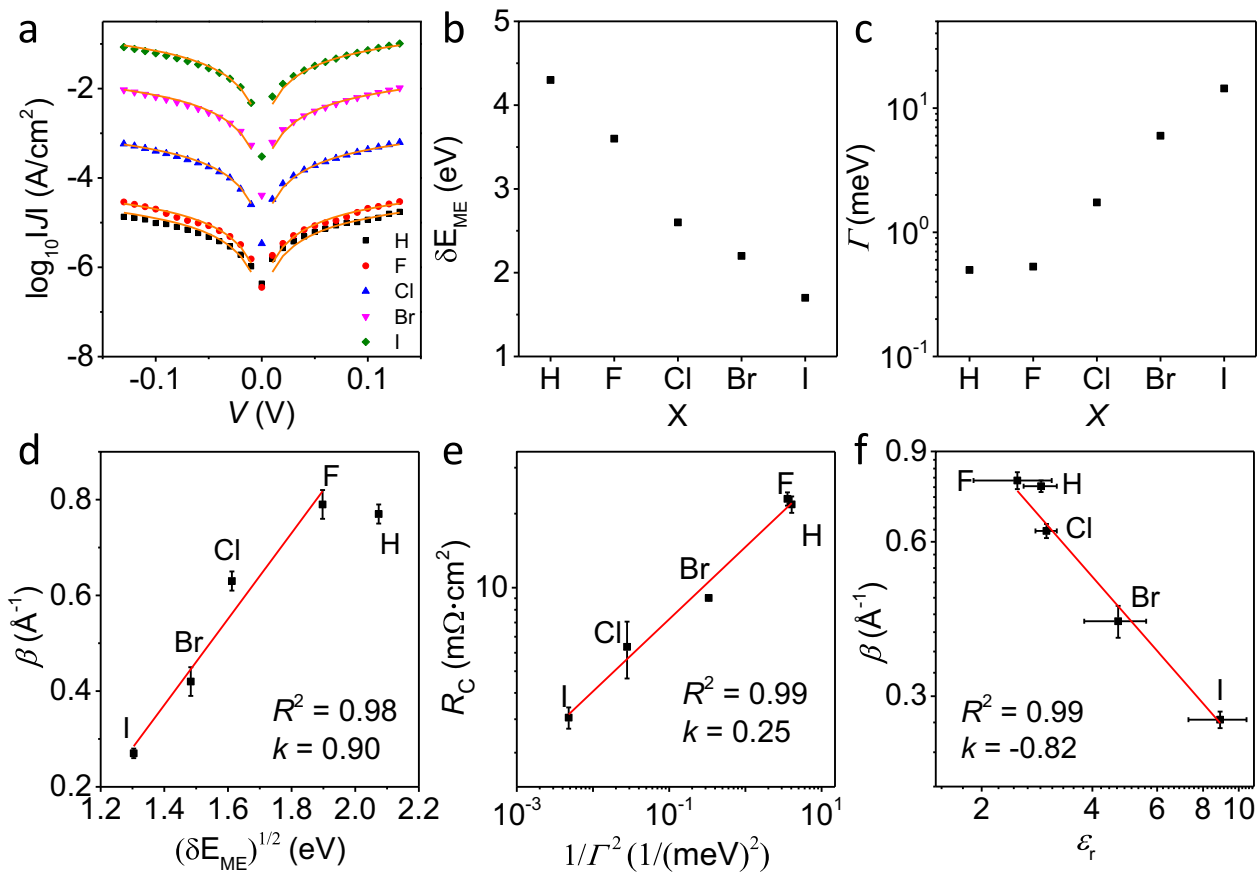

Fig. 6 Single-level Landauer model analysis. a The modelled current through the $\mathrm{Ag}-\mathrm{S}\left(\mathrm{CH}_{2}\right)_{14} \mathrm{X} / / \mathrm{GaO}_{x} / \mathrm{EGaln}$ junctions using Landauer theory (orange solid lines are Landauer fits, symbols represent experimental data). The values of tunnelling barrier height $\left(\delta E_{\mathrm{ME}}\right)(\mathbf{b})$ and the coupling strength $(\Gamma)$ (c) used for modelling the current through the junctions. $\mathbf{d}$ Tunnelling decay coefficient $\beta$ vs. $\sqrt{\delta E_{M E}}$ with a linear fit (red line), the error bars represent the standard deviations of the $\beta$ values from linear fits to Eq. (1). e Double-log plot of $R_{\mathrm{C}}$ vs. $1 / \Gamma^{2}$ ( $R_{\mathrm{C}}$ represents contact resistance) where the red line is a power-law fit with a slope of 0.25 and $R^{2}=0.99$, error bars of $R_{C}$ represent the standard deviations of three independent measurements. $\mathbf{f}$ Double-log plot of $\beta$ vs. $\varepsilon_{r}$ where the red line is a fit with a slope of -0.82 and $R^{2}=0.99$. The error bars of $\beta$ represents the same as panel $\mathbf{b}$, and of $\varepsilon_{\mathrm{r}}$ represent the standard deviations of three independent measurements.

of fittings were done for each $\mathrm{X}$, fixing $\delta E_{\mathrm{ME}}$ to the values extracted from DFT and leaving $\gamma_{\mathrm{L}}, \gamma_{\mathrm{R}}, \eta$ and $\sigma$ as fitting parameters to obtain best fits to the data of junctions of $\mathrm{Ag}-\mathrm{S}$ $\left(\mathrm{CH}_{2}\right)_{14} \mathrm{X} / / \mathrm{GaO}_{x} / \mathrm{EGaIn}$ (Supplementary Table 12). Figure 6a shows the fits of the theoretical model (orange lines) to the experimental data (symbols) for each $\mathrm{S}\left(\mathrm{CH}_{2}\right)_{14} \mathrm{X}$ molecule. Figure $6 \mathrm{~b}, \mathrm{c}$ shows the two parameters that vary across molecules: the energy $\delta E_{\mathrm{ME}}$ of the frontier orbital (extracted from DFT), which decreases from 4.3 to $1.7 \mathrm{eV}$ on moving through the sequence $\mathrm{H}-\mathrm{F}-\mathrm{Cl}-\mathrm{Br}-\mathrm{I}$ (Fig. $6 \mathrm{~b}$ ), and the overall tunnelling rate through the junction (i.e., the $\Gamma$ ), defined as $\Gamma=\frac{\gamma_{\mathrm{L}} \gamma_{\mathrm{R}}}{\gamma_{\mathrm{L}}+\gamma_{\mathrm{R}}}$, which increases exponentially along the halogen sequence (Fig. $6 c$ ) and accounts for the observed exponential increase of the current through the junctions.

Figure $6 \mathrm{~d}$ shows a linear relationship between calculated $\sqrt{\delta E_{\mathrm{ME}}}$ and measured $\beta$, which agrees with commonly used coherent tunnelling models ${ }^{2,5,18}$ including the Simmons model which also accounts for $\varepsilon_{\mathrm{r}}$. However, the Simmons model also predicts a decrease of the tunnelling rates with increasing $\varepsilon_{\mathrm{r}}$ due to a reduction of the image charge effects in the electrodes due to screening within the SAM ${ }^{18,66}$. This reduction of image charge in effect increases $\delta E_{\mathrm{ME}}$ and, consequently, $\beta$, but we observe the opposite trend. Using the same model, a reduction of the effective electron mass could also account for an increase in tunnelling rates, but it is not clear how the effective electron mass would change as a function of $\mathrm{X}$ with the essentially localized features derived from the HOMO and LUMO. Furthermore, Vilan ${ }^{67}$ argued that changes in the electron mass are equivalent to changes in the $\delta E_{\mathrm{ME}}$ within the Simmons model, which further complicates the interpretation of our findings within this framework.

The experimentally determined values of $R_{\mathrm{C}}$ have been related to $\Gamma$ as $R_{\mathrm{C}} \propto \Gamma^{-2}$ (ref. ${ }^{30}$ ), i.e., the coupling of the molecules with the electrode we have determined above (Fig. 6e). To test whether this holds for the SAMs studied here, Fig. 6e shows a double-log plot of $R_{\mathrm{C}}$ vs. $1 / \Gamma^{2}$, indicating that our results can be explained, at least qualitatively, using this picture: changes in both $\delta E_{\mathrm{ME}}$ and $\Gamma$ can lower $\beta$, in accordance with findings by other ${ }^{27-35}$. This approach, however, does not capture the observed changes in the dielectric response of the junctions directly, and, of course, it does not explicitly account for the local changes in the electrostatic potential profile induced by X observed in the DFT calculations; these effects are essentially compensated by the large change in $\Gamma$ of 29 times.

An interesting finding was reported by Berlin and Ratner ${ }^{68}$ based on an alternative model to describe tunnelling across barriers with charge traps, with the finding that $\beta \propto 1 / \sqrt{\varepsilon_{r}}$. In this framework, the distance dependence of the conductance is related to a thicknessdependent barrier akin to the one inherent to the Simmons model ${ }^{67,69}$. In their model, however, the barrier arises from the presence of localized charge traps along the path of charge migration leading to a non-linear potential drop between the macroscopic leads. Figure $6 \mathrm{f}$ shows the linear relation of the double-log plot of $\beta$ vs. $\varepsilon_{\mathrm{r}}$ with a slope of -0.82 which is lower than the expected -0.5 from the model by Berlin et al. ${ }^{68}$, but note that a change in the contact resistance or further changes in the barrier shape are not taken into consideration in this model. In our experiments, however, the contact resistance changes and our DFT calculations show that the barrier shape at the SAM//top electrode interface is affected by X.

\section{Discussion}

This work shows that substitution of a single highly polarizable atom can have a pronounced effect on the energy level alignment, charge transport rate, and dielectric response of molecular junctions. We were able to tune $\beta$ over a wide range from 0.25 to $0.75 \AA^{-1}$ across 
saturated alkyl chains by changing one atom per molecule inside large-area $\left(\sim 350 \mu \mathrm{m}^{2}\right)^{52}$ junctions. The largest effects of $\mathrm{X}$ on $J$ are found in the longest molecules of $\mathrm{S}\left(\mathrm{CH}_{2}\right)_{18} \mathrm{X}$ where the $J$ increases by a factor of $10^{\approx 5}$ when $\mathrm{X}$ changes from $\mathrm{H}$ to $\mathrm{I}$. In contrast, for the shortest molecule $\mathrm{S}\left(\mathrm{CH}_{2}\right)_{10} \mathrm{X}, J$ increases by a factor of $10^{\approx 2}$, indicating that the observed changes in $J$, and the corresponding values of $\beta$, are driven by more than just changes in the interfaces, which has been discussed previously by Frisbie and co-workers ${ }^{9}, 38$. Combining experiment with DFT and Landauer charge transport models, we established three factors that contribute to the dramatic change in $\beta$ of these aliphatic halogenated junctions with varying $\mathrm{X}$ and associated increase in $\alpha$ and $\varepsilon_{\mathrm{r}}$ : (1) The HOMO-LUMO gap ${ }^{5,45}$ and associated $\delta E_{\mathrm{ME}}$ is reduced which lowers $\beta$, (2) the shape of the tunnelling barrier is modified at the SAM-top electrode interface, resulting in larger potential drops at this interface, and (3) the electronic coupling $\Gamma$ of the molecular orbitals with the electrodes increases (potentially because of an increase in the van der Waals interactions along the halogen series).

In a broad context of widely used charge tunnelling mechanisms, our findings point out their limitations highlighting the need for improved models that take dielectric (or collective) effects of the junctions into consideration. Specifically, the popular Simmons model predicts that image charge effects in the electrodes are reduced with increasing $\varepsilon_{\mathrm{r}}$, resulting in lowering of the tunnelling rates ${ }^{67}$, which is in sharp contrast to what we find. Superexchange models ${ }^{19-21}$ also fail to explain our observations, since the coupling between the molecular repeat unit (i.e., the $\mathrm{CH}_{2}$ units) was not changed here. Conversely, the Landauer model ${ }^{65}$ could explain our results at least qualitatively, but not quantitatively. This is because it does not treat electrostatic effects in the junctions explicitly and selfconsistently which resulted in our case in a large increase in the values of $\Gamma$ (29 times) even though the $R_{\mathrm{C}}$ only changed by a factor of five in our experiments. Interestingly, a mechanism proposed by Berlin and Ratner ${ }^{68}$ that is based on charge traps provides a hypothesis for how the value of $\beta$ could decrease with increasing $\varepsilon_{\mathrm{r}}$. Although the physical interpretation differs as the tunnelling behaviour is explained in terms of charge traps rather than the electrostatic response of the SAM inside the junction, this line of thought stimulates further theoretical and experimental testing of the presence of "impurities" - here in the form of polarizable atoms-as charge carriers that move across the energy band profiles. Although our findings suggest a correlation between $\beta$ and $\varepsilon_{\mathrm{r}}$, the increase of $\varepsilon_{\mathrm{r}}$ as a function of $\mathrm{X}$ could not be reproduced in our DFT calculations, which may be because the calculations do not take the SAM-metal interface into consideration, and perhaps other factors are important such as (partial) charging of highly polarizable molecules during charge transport inside the junctions. To summarize, our work proposes an effective way of tuning the tunnelling rates across molecular junctions without chemically altering the backbone of the molecules and highlights the importance of understanding dielectric effects in these junctions. We hope that our findings will stimulate further experimental and theoretical investigations towards establishing improved transport mechanisms for junctions in their in situ physicochemical environment and electronic states inside working devices.

\section{Data availability}

The data that support the findings of this study are available within the article and Supplementary Information file, or at https://doi.org/10.7910/DVN/WZAIGU. Source data are provided with this paper.

\section{Code availability}

The code for the analysis of $J(V)$ results in this study is available at https://doi.org/ 10.7910/DVN/WZAIGU.
Received: 31 August 2020; Accepted: 30 April 2021; Published online: 08 June 2021

\section{References}

1. Shah, A. et al. Electron transfer in peptides. Chem. Soc. Rev. 44, 1015-1027 (2015).

2. Vilan, A., Aswal, D. \& Cahen, D. Large-area, ensemble molecular electronics: motivation and challenges. Chem. Rev. 117, 4248-4286 (2017).

3. Jortner, J., Nitzan, A. \& Ratner, M. A. in Introducing Molecular Electronics (eds. Cuniberti, G. et al.) 13-54 (Springer, 2005).

4. Jia, C. \& Guo, X. Molecule-electrode interfaces in molecular electronic devices. Chem. Soc. Rev. 42, 5642-5660 (2013).

5. Metzger, R. M. Unimolecular electronics. Chem. Rev. 115, 5056-5115 (2015).

6. Jeong, H., Kim, D., Xiang, D. \& Lee, T. High-yield functional molecular electronic devices. ACS Nano 11, 6511-6548 (2017).

7. Hylke, B. A. \& de Bert, B. Electrical conduction through single molecules and self-assembled monolayers. J. Phys. Condens. Matter 20, 013001 (2008).

8. Nitzan, A. Electron transmission through molecules and molecular interfaces. Annu. Rev. Phys. Chem. 52, 681-750 (2001).

9. Xie, Z., Bâldea, I. \& Frisbie, C. D. Determination of energy-level alignment in molecular tunnel junctions by transport and spectroscopy: self-consistency for the case of oligophenylene thiols and dithiols on $\mathrm{Ag}, \mathrm{Au}$, and Pt electrodes. J. Am. Chem. Soc. 141, 3670-3681 (2019).

10. Kumar, K. S., Neoh, E. H. L., Vilan, A. \& Nijhuis, C. A. Protective layers based on carbon paint to yield high-quality large-area molecular junctions with low contact resistance. J. Am. Chem. Soc. 142, 3513-3524 (2020).

11. Simeone, F. C. et al. Defining the value of injection current and effective electrical contact area for EGaIn-based molecular tunneling junctions. J. Am. Chem. Soc. 135, 18131-18144 (2013).

12. Karuppannan, S. K., Pasula, R. R., Lim, S. \& Nijhuis, C. A. Long-range tunneling processes across ferritin-based junctions. Adv. Mater. 28, 1824-1830 (2016)

13. Christopher, D. B. et al. Protein bioelectronics: a review of what we do and do not know. Rep. Prog. Phys. 81, 026601 (2018).

14. Baghbanzadeh, M. et al. Charge tunneling along short oligoglycine chains. Angew. Chem. Int. Ed. 54, 14743-14747 (2015).

15. Baghbanzadeh, M. et al. Anomalously rapid tunneling: charge transport across self-assembled monolayers of oligo(ethylene glycol). J. Am. Chem. Soc. 139, 7624-7631 (2017)

16. Su, T. A., Li, H., Steigerwald, M. L., Venkataraman, L. \& Nuckolls, C. Stereoelectronic switching in single-molecule junctions. Nat. Chem. 7, 215-220 (2015).

17. Yan, H. et al. Activationless charge transport across 4.5 to $22 \mathrm{~nm}$ in molecular electronic junctions. Proc. Natl Acad. Sci. USA 110, 5326-5330 (2013).

18. Simmons, J. G. Generalized formula for the electric tunnel effect between similar electrodes separated by a thin insulating film. J. Appl. Phys. 34, 1793-1803 (1963)

19. Naleway, C. A., Curtiss, L. A. \& Miller, J. R. Superexchange-pathway model for long-distance electronic couplings. J. Phys. Chem. 95, 8434-8437 (1991).

20. McConnell, H. M. Intramolecular charge transfer in aromatic free radicals. J. Chem. Phys. 35, 508-515 (1961)

21. Todd, M. D., Nitzan, A. \& Ratner, M. A. Electron transfer via superexchange: a time-dependent approach. J. Phys. Chem. 97, 29-33 (1993).

22. Juhaniewicz, J. \& Sek, S. Peptide molecular junctions: distance dependent electron transmission through oligoprolines. Bioelectrochemistry 87, 21-27 (2012).

23. Beratan, D. N. et al. Charge transfer in dynamical biosystems, or the treachery of (static) images. Acc. Chem. Res. 48, 474-481 (2015).

24. Zhang, Y., Liu, C., Balaeff, A., Skourtis, S. S. \& Beratan, D. N. Biological charge transfer via flickering resonance. Proc. Natl Acad. Sci. USA 111, 10049-10054 (2014).

25. Berlin, Y. A., Kurnikov, I. V., Beratan, D., Ratner, M. A. \& Burin, A. L. in Long-Range Charge Transfer in DNA II (ed. Schuster, G. B.) 1-36 (Springer, 2004).

26. Amdursky, N. et al. Electronic transport via proteins. Adv. Mater. 26, 7142-7161 (2014).

27. Sangeeth, C. S. S. et al. Comparison of DC and AC transport in 1.5-7.5 nm oligophenylene imine molecular wires across two junction platforms: eutectic Ga-In versus conducting probe atomic force microscope junctions. J. Am. Chem. Soc. 138, 7305-7314 (2016).

28. Nguyen, Q. V. et al. Highly efficient long-range electron transport in a viologen-based molecular junction. J. Am. Chem. Soc. 140, 10131-10134 (2018).

29. Sepunaru, L. et al. Electronic transport via homopeptides: the role of side chains and secondary structure. J. Am. Chem. Soc. 137, 9617-9626 (2015). 
30. Xie, Z., Bâldea, I., Smith, C. E., Wu, Y. \& Frisbie, C. D. Experimental and theoretical analysis of nanotransport in oligophenylene dithiol junctions as a function of molecular length and contact work function. ACS Nano 9, 8022-8036 (2015).

31. Gunasekaran, S. et al. Near length-independent conductance in polymethine molecular wires. Nano Lett. 18, 6387-6391 (2018).

32. Sedghi, G. et al. Long-range electron tunnelling in oligo-porphyrin molecular wires. Nat. Nanotechnol. 6, 517-523 (2011).

33. Leary, E. et al. Bias-driven conductance increase with length in porphyrin tapes. J. Am. Chem. Soc. 140, 12877-12883 (2018).

34. Zhang, Y. et al. Tunneling probability increases with distance in junctions comprising self-assembled monolayers of oligothiophenes. J. Am. Chem. Soc. 140, 15048-15055 (2018).

35. Algethami, N., Sadeghi, H., Sangtarash, S. \& Lambert, C. J. The conductance of porphyrin-based molecular nanowires increases with length. Nano Lett. 18, 4482-4486 (2018).

36. Sangtarash, S. et al. Gateway state-mediated, long-range tunnelling in molecular wires. Nanoscale 10, 3060-3067 (2018)

37. Engelkes, V. B., Beebe, J. M. \& Frisbie, C. D. Length-dependent transport in molecular junctions based on SAMs of alkanethiols and alkanedithiols: effect of metal work function and applied bias on tunneling efficiency and contact resistance. J. Am. Chem. Soc. 126, 14287-14296 (2004).

38. Xie, Z., Bâldea, I. \& Frisbie, C. D. Energy level alignment in molecular tunnel junctions by transport and spectroscopy: self-consistency for the case of alkyl thiols and dithiols on Ag, Au, and Pt electrodes. J. Am. Chem. Soc. 141, 18182-18192 (2019).

39. Xie, Z., Bâldea, I. \& Frisbie, C. D. Why one can expect large rectification in molecular junctions based on alkane monothiols and why rectification is so modest. Chem. Sci. 9, 4456-4467 (2018).

40. Gu, M. W., Peng, H. H., Chen, I. P. \& Chen, C. H. Tuning surface d bands with bimetallic electrodes to facilitate electron transport across molecular junctions. Nat. Mater. 20, 658-664 (2021).

41. Sangeeth, C. S. S., Wan, A. \& Nijhuis, C. A. Equivalent circuits of a selfassembled monolayer-based tunnel junction determined by impedance spectroscopy. J. Am. Chem. Soc. 136, 11134-11144 (2014).

42. Van Dyck, C., Marks, T. J. \& Ratner, M. A. Chain length dependence of the dielectric constant and polarizability in conjugated organic thin films. ACS Nano 11, 5970-5981 (2017).

43. Natan, A., Kuritz, N. \& Kronik, L. Polarizability, susceptibility, and dielectric constant of nanometer-scale molecular films: a microscopic view. Adv. Funct. Mater. 20, 2077-2084 (2010).

44. Romaner, L., Heimel, G., Ambrosch-Draxl, C. \& Zojer, E. The dielectric constant of self-assembled monolayers. Adv. Funct. Mater. 18, 3999-4006 (2008).

45. Wang, D. et al. Tuning the tunneling rate and dielectric response of SAMbased junctions via a single polarizable atom. Adv. Mater. 27, 6689-6695 (2015).

46. Haj-Yahia, A.-E. et al. Substituent variation drives metal/monolayer/ semiconductor junctions from strongly rectifying to ohmic behavior. $A d v$. Mater. 25, 702-706 (2013)

47. Baghbanzadeh, M., Pieters, P. F., Yuan, L., Collison, D. \& Whitesides, G. M. The rate of charge tunneling in EGaIn junctions is not sensitive to halogen substituents at the self-assembled monolayer// $\mathrm{Ga}_{2} \mathrm{O}_{3}$ interface. ACS Nano 12, 10221-10230 (2018).

48. Stone, A. J. The Theory of Intermolecular Forces (Oxford University Press, 2013).

49. Yoon, H. J. et al. The rate of charge tunneling through self-assembled monolayers is insensitive to many functional group substitutions. Angew. Chem. Int. Ed. 51, 4658-4661 (2012).

50. Yoon, H. J., Bowers, C. M., Baghbanzadeh, M. \& Whitesides, G. M. The rate of charge tunneling is insensitive to polar terminal groups in self-assembled monolayers in $\mathrm{Ag}^{\mathrm{TS}} \mathrm{S}\left(\mathrm{CH}_{2}\right)_{\mathrm{n}} \mathrm{M}\left(\mathrm{CH}_{2}\right)_{\mathrm{m}} \mathrm{T} / / \mathrm{Ga}_{2} \mathrm{O}_{3} / \mathrm{EGaIn}$ junctions. J. Am. Chem. Soc. 136, 16-19 (2014).

51. Bowers, C. M. et al. Introducing ionic and/or hydrogen bonds into the SAM// $\mathrm{Ga}_{2} \mathrm{O}_{3}$ top-interface of $\mathrm{Ag}^{\mathrm{TS}} / \mathrm{S}\left(\mathrm{CH}_{2}\right)_{\mathrm{n}} \mathrm{T} / / \mathrm{Ga}_{2} \mathrm{O}_{3} / \mathrm{EGaIn}$ junctions. Nano Lett. 14, 3521-3526 (2014).

52. Chen, X., Hu, H., Trasobares, J. \& Nijhuis, C. A. Rectification ratio and tunneling decay coefficient depend on the contact geometry revealed by in situ imaging of the formation of EGaIn junctions. ACS Appl. Mater. Inter. 11 21018-21029 (2019).

53. Nerngchamnong, N. et al. The role of van der Waals forces in the performance of molecular diodes. Nat. Nanotechnol. 8, 113-118 (2013).

54. Wang, W., Lee, T. \& Reed, M. A. Mechanism of electron conduction in selfassembled alkanethiol monolayer devices. Phys. Rev. B 68, 035416 (2003)

55. Ashcroft, N. W. \& Mermin, N. D. Solid State Physics (Holt, Rinehart and Winston, 1976).

56. Kresse, G. \& Furthmüller, J. Efficient iterative schemes for ab initio total-energy calculations using a plane-wave basis set. Phys. Rev. B 54, 11169-11186 (1996).
57. Lee, H. J., Jamison, A. C. \& Lee, T. R. Surface dipoles: a growing body of evidence supports their impact and importance. Acc. Chem. Res. 48, 3007-3015 (2015)

58. Chen, X. et al. Interplay of collective electrostatic effects and level alignment dictates the tunneling rates across halogenated aromatic monolayer junctions. J. Phys. Chem. Lett. 10, 4142-4147 (2019).

59. Abu-Husein, T. et al. The effects of embedded dipoles in aromatic selfassembled monolayers. Adv. Funct. Mater. 25, 3943-3957 (2015).

60. Jiang, L., Sangeeth, C. S. S., Yuan, L., Thompson, D. \& Nijhuis, C. A. Onenanometer thin monolayers remove the deleterious effect of substrate defects in molecular tunnel junctions. Nano Lett. 15, 6643-6649 (2015).

61. Yuan, L., Jiang, L. \& Nijhuis, C. A. The drive force of electrical breakdown of large-area molecular tunnel junctions. Adv. Funct. Mater. 28, 1801710 (2018).

62. Heitzer, H. M., Marks, T. J. \& Ratner, M. A. First-principles calculation of dielectric response in molecule-based materials. J. Am. Chem. Soc. 135, 9753-9759 (2013).

63. Rozhkov, I. N., Raevsky, A. M., Gushchin, S. I., Ignatova, J. L. \& Semin, G. K. Evaluation of charge changes on chlorine, bromine and iodine atoms in perfluoroalkyl halogenides based on nuclear quadrupole resonance data. J. Fluor. Chem. 58, 234-234 (1992).

64. Probst, A., Raab, K., Ulm, K. \& von Werner, K. Synthesis and chemistry of perfluoro-2-iodo-2-methyl-alkanes. J. Fluor. Chem. 37, 223-245 (1987).

65. Garrigues, A. R. et al. A single-level tunnel model to account for electrical transport through single molecule- and self-assembled monolayer-based junctions. Sci. Rep. 6, 26517 (2016)

66. Simmons, J. G. Electric tunnel effect between dissimilar electrodes separated by a thin insulating film. J. Appl. Phys. 34, 2581-2590 (1963).

67. Vilan, A. Analyzing molecular current-voltage characteristics with the simmons tunneling model: scaling and linearization. J. Phys. Chem. C 111, 4431-4444 (2007)

68. Berlin, Y. A. \& Ratner, M. A. Conduction of metal-thin organic film-metal junctions at low bias. J. Phys. Chem. C 122, 7557-7563 (2018).

69. Karuppannan, S. K., Hu, H., Troadec, C., Vilan, A. \& Nijhuis, C. A. Ultrasmooth and photoresist-free micropore-based EGaIn molecular junctions: fabrication and how roughness determines voltage response. $A d v$. Funct. Mater. 29, 1904452 (2019).

\section{Acknowledgements}

We acknowledge fruitful discussions with Ayelet Vilan (Weizmann Institute of Science) and Gemma Solomon (University of Copenhagen). Fundings by the Ministry of Education (MOE) for supporting this research under award No. MOE2019-T2-1-137 and R-143-000-B30-112 are acknowledged. Prime Minister's Office, Singapore under its Medium sized centre programme is also acknowledged for supporting this research. The authors would furthermore like to acknowledge the Singapore Synchrotron Light Source (SSLS) for providing the facilities at the Surface, Interface and Nanostructure Science (SINS) beam line under NUS core support C-380-003-003-001. The Laboratory is a National Research Infrastructure under the National Research Foundation Singapore We moreover acknowledge funding from the Alexander von Humboldt Foundation within the framework of the Sofja Kovalevskaja Award, endowed by the German Federal Ministry of and Research, and the Technical University of Munich-Institute for Advanced Study, funded by the German Excellence Initiative and the European Union Seventh Framework Programme under Grant Agreement No. 291763. D.T. thanks Science Foundation Ireland (SFI) for support (awards Grant Numbers 15/CDA/3491 and 12/RC/2275_P2) and for computing resources at the SFI/Higher Education Authority Irish Centre for High-End Computing (ICHEC). Finally, we also acknowledge support from the U.S. National Science Foundation (Grant No. ECCS\#1916874). The authors gratefully acknowledge the Gauss Centre for Supercomputing e.V. (www.gauss-centre.eu) for funding this project by providing computing time through the John von Neumann Institute for Computing (NIC) on the GCS Supercomputer JUWELS at Jülich Supercomputing Centre (JSC).

\section{Author contributions}

X.C., D.A.E., and C.A.N. conceived and designed the project. X.C. synthesized the compounds, performed electrical characterizations, and associated data analysis; B.K. and D.A.E. performed the DFT calculations; F.A., C.N., and E.d.B. performed the Landauer model analysis; X.C. and X.Y. conducted the XPS and UPS measurements; D.T. conducted the molecular dynamics; all the authors discussed the results and prepared the manuscript.

\section{Competing interests}

The authors declare no competing interests. 


\section{Additional information}

Supplementary information The online version contains supplementary material available at https://doi.org/10.1038/s41467-021-23528-8.

Correspondence and requests for materials should be addressed to D.A.E. or C.A.N.

Peer review information Nature Communications thanks the anonymous reviewer(s) for their contribution to the peer review of this work. Peer reviewer reports are available.

Reprints and permission information is available at http://www.nature.com/reprints

Publisher's note Springer Nature remains neutral with regard to jurisdictional claims in published maps and institutional affiliations. (c) (1) Open Access This article is licensed under a Creative Commons Attribution 4.0 International License, which permits use, sharing, adaptation, distribution and reproduction in any medium or format, as long as you give appropriate credit to the original author(s) and the source, provide a link to the Creative Commons license, and indicate if changes were made. The images or other third party material in this article are included in the article's Creative Commons license, unless indicated otherwise in a credit line to the material. If material is not included in the article's Creative Commons license and your intended use is not permitted by statutory regulation or exceeds the permitted use, you will need to obtain permission directly from the copyright holder. To view a copy of this license, visit http://creativecommons.org/ licenses/by/4.0/.

(C) The Author(s) 2021 